\title{
EchoGéo
}

$51 \mid 2020$

Territoires « cyclonés »

\section{Adaptation aux cyclones et au risque de submersion marine dans les communes littorales de Guadeloupe}

Michel Desse, Monique Gherardi et Simon Charrier

\section{(2) OpenEdition}

Journals

Édition électronique

URL : https://journals.openedition.org/echogeo/19207

DOI : 10.4000/echogeo.19207

ISSN : 1963-1197

Éditeur

Pôle de recherche pour l'organisation et la diffusion de l'information géographique (CNRS UMR 8586)

Référence électronique

Michel Desse, Monique Gherardi et Simon Charrier, « Adaptation aux cyclones et au risque de submersion marine dans les communes littorales de Guadeloupe », EchoGéo [En ligne], 51 | 2020, mis en ligne le 27 mai 2020, consulté le 11 août 2021. URL : http://journals.openedition.org/echogeo/ 19207 ; DOl : https://doi.org/10.4000/echogeo.19207

Ce document a été généré automatiquement le 11 août 2021.

EchoGéo est mis à disposition selon les termes de la licence Creative Commons Attribution - Pas d'Utilisation Commerciale - Pas de Modification 4.0 International (CC BY-NC-ND) 


\title{
Adaptation aux cyclones et au risque de submersion marine dans les communes littorales de Guadeloupe
}

\author{
Michel Desse, Monique Gherardi et Simon Charrier
}

"Quelle importance sociale doit-on donner aux risques naturels? Comment construire intellectuellement un système de risques qui prenne en compte à la fois les risques auxquels une société est soumise et tous les avantages recherchés qui leurs sont associés?».

Ce questionnement proposé par Hervé Franquart (2016) est au cœur de la problématique des littoraux guadeloupéens qui devraient connaître d'ici 2100 une remontée de la mer estimée entre 40 et $60 \mathrm{~cm}$ auxquels se rajouteront épisodiquement des surcotes cycloniques pouvant atteindre localement 3 voir $5 \mathrm{~m}$. Les territoires affectés présentent de très fortes densités souvent supérieures à la moyenne insulaire $\left(253 \mathrm{hab} / \mathrm{km}^{2}\right)$, du fait de la présence des terrains plats qui attirent les populations et les entreprises (Pagney Bénito-Espinal, 1998). La Guadeloupe connaît un accroissement continu de sa population dans les bourgs littoraux depuis 1848, qui s'est renforcé dans les années 1950 dans un contexte d'abandon de l'agriculture vivrière et caféière sur la côte-sous-le-vent et depuis les années 1980 avec le déclin de la monoculture de la canne à sucre en Grande-Terre. Parallèlement les autres activités traditionnelles comme la petite pêche, l'agriculture vivrière et, plus récemment, la filière de la banane périclitent au grès des aléas climatiques (tempêtes tropicales, cyclones, mais aussi épisodes de sécheresse), des politiques agricoles de l'UE, ou à l'occasion de ruptures liées aux crises économiques ou environnementales. Dans ces conditions, le développement du secteur touristique et des loisirs qui génère des emplois accroît l'attractivité des bourgs littoraux qui demeuraient essentiellement tournés vers la pêche et quelques fonctions d'encadrement (Desse, 2011). Dans le même temps, avec l'accentuation des politiques de rattrapage dans le cadre de la départementalisation, puis avec les différentes politiques régionales d'aménagement, ces bourgs connaissent 
des mesures de résorption de l'habitat insalubre (RHI) pour améliorer les conditions d'habitat ou de relocalisation des populations sinistrées à la suite du passage des différents cyclones qui affectent l'île ${ }^{1}$ (Pagney Bénito-Espinal, 2002). La violence et les dégâts occasionnés par le passage de Hugo en 1989 ont constitué un événement fondateur et transformé le regard des élus sur le littoral. Le triptyque case de pêcheur, canot sur la plage et la mer, véritable lieu de vie du méritoire traditionnel devient peu à peu pour les collectivités, une marge qu'il convient de se réapproprier et d'aménager pour limiter les risques et les dégâts occasionnés par les houles cycloniques.

2 À priori, ces réaménagements précurseurs aux zones établies après Xynthia en métropole apparaissent comme des formes de résilience des communautés littorales et des collectivités locales.

\section{Les cyclones accélérateurs des ruptures économiques et sociales}

3 Le passage d'un cyclone constitue un choc pour les habitants et leur support de vie économique et social, entraînant des ruptures sociétales et économiques. Passées les premières semaines, les choix de reconstruction s'imposent (Lassailly-Jacob et Desse, 2010). Au travers de deux exemples touchant les mêmes sociétés insulaires, nous analyserons les stratégies positives et proactives ou au contraire les paralysies par manque de moyens et de stratégie prospective dans un contexte de crise mondiale.

4 En 1989, le cyclone Hugo a ravagé la Guadeloupe et en particulier la Grande-Terre. Le cyclone a entraîné la mort d'une dizaine de personnes. Les dégâts furent énormes et sont estimés à 4 milliards de francs affectant un grand nombre de cases en bois des ouvriers agricoles et des plus démunis qui n'ont pas résisté en campagne comme dans les bourgs (21 000 sans-abris).

5 Les secteurs économiques ont aussi été très touchés. En effet, les vents ont détruit la quasi-totalité des cultures maraîchères, les bananeraies de la Basse-Terre et les champs de cannes à sucre qui constituaient une quasi-monoculture en Grande-Terre causant, pour 466 millions de francs de dommages. Ce système reposant sur les cultures d'exportation montrait ses limites et bénéficiait depuis la fin des années 1960, de subventions par les collectivités territoriales, l'État et l'Union Européenne. Tous les indicateurs montraient un modèle agricole à bout de souffle dans un contexte de chômage grandissant: la concurrence du sucre cubain et brésilien, l'échec de la réforme foncière qui offrait des petites propriétés juste rentables en 1960, mais qui ne permettait plus d'attirer les jeunes exploitants à la fin des années 1980. Les secteurs du tourisme sont aussi atteints pour un montant estimé à 152 millions de francs, soit un coût total des réparations qui atteint les 610 millions d'euros (Desse, 2013).

6 Quelques mois plus tard pour les uns, quelques années pour d'autres, les aides de l'État et les financements multiples ont finalement profité à de nombreux Guadeloupéens qui ont remplacé leurs cases sinistrées par des villas en brique couvertes d'une dalle en béton et très vite surmontée par un toit en tôle. Dans le domaine agricole, une réflexion de fond a permis de tourner le dos à la monoculture de la canne pour des productions plus rentables comme le melon vendu en métropole en hiver ou le maraîchage. Les soles cannières qui demeurent sont centrées autour de l'unique centrale sucrière, toujours subventionnée mais qui opère une reconversion afin de mieux valoriser sa 
production pour le tourisme et l'hôtellerie par exemple. Les pêcheurs qui ont subi d'importants dégâts (perte des canots, des engins de pêche, des installations à terre ont aussi été indemnisés entrainant le passage du canot traditionnel en bois, faiblement motorisé, au canot en polyester à coque semi-planante doté d'une forte motorisation. À terre, les installations regroupées autours de petits ports de pêche artisanale bénéficient de machines à glace, d'étals de vente aux normes européennes, de locaux pour les marins-pêcheurs.

7 Le secteur du tourisme, déjà dynamisé par les lois de défiscalisation de 1986, est aussi aidé, permettant la modernisation de certains hôtels internationaux construits depuis une quinzaine d'années. Surtout, les financements ont permis l'essor des services touristiques auprès de la population locale. Les subventions ont participé à l'appropriation de l'économie touristique et la diffusion des chambres d'hôtes rendue possible par l'amélioration de l'habitat et la hausse du niveau de confort. On assiste à la multiplication des petits complexes de location de bungalows, des sociétés de location de voiture, d'engins de plage ou de découverte de l'île en bateau, en plongée... Les dégâts occasionnés aux ports de plaisance du Bas du Fort et de Saint-François ont aussi permis des réparations et des agrandissements qui ont attiré les compagnies de locations de voiliers de plaisance déjà intéressées par les lois de défiscalisation. Au-delà du traumatisme qui marque durablement les habitants, Hugo a donc eu des retombées bénéfiques parce que déjà la société guadeloupéenne et les collectivités territoriales renforcées par les lois de décentralisation de 1981 amorçaient une dynamique économique positive. Le cyclone a finalement permis une certaine rupture accélérée par un fort investissement de l'État et de l'Union européenne.

Quand les îles traversent des conjonctures économiques et sociales dégradées, l'impact du cyclone est plus profond, entrainant des ruptures régressives qui débouchent sur de véritables crises sociales et politiques parce que les sociétés et les économies n'arrivent pas à se remettre du choc initial. Ce fut le cas avec le cyclone Dean (vents à $180 \mathrm{~km} / \mathrm{h}$ ) qui a ravagé la Martinique et la Guadeloupe le 17 août 2007, entraînant la destruction des cultures maraîchères et vivrières, des plantations de bananiers (50000 ha à la Martinique). En mer, les nasses, les filets et les DCP, dispositifs de concentration de poisson ${ }^{2}$ mouillés au large, sont perdus. Le cyclone a aussi réduit de $10 \%$ la production du secteur secondaire et touche directement la petite hôtellerie qui ne peut faire face à la diminution de la clientèle durant plusieurs semaines. En 2007, la conjoncture internationale a changé et surtout, la très forte augmentation du prix du pétrole rappelle les contraintes de l'insularité qui semblaient être dépassées par les progrès de l'internet, la multiplication des compagnies aériennes et l'essor du trafic par conteneur qui offre davantage de flexibilité pour les productions agricoles locales et notamment la banane (Desse, 2013).

9 En 2007, le secteur touristique est largement en crise dans les Antilles françaises qui subissent la concurrence de Cuba et de Saint-Domingue offrant une hôtellerie de meilleure qualité, et répondant aux nouvelles attentes de la clientèle à des prix plus économiques. De nombreux hôtels sont fermés et des friches hôtelières apparaissent même dans des zones hôtelières prestigieuses (Marina du François en Guadeloupe, Pointe du Bout à la Martinique, Saint-Martin). Le secteur de la croisière est aussi en difficulté puisque depuis les attentats de 2001, les compagnies de croisières préfèrent se recentrer sur les îles anglophones, entraînant encore une forte diminution de cette activité. Dans le secteur agricole, les espoirs engendrés par la diversification des 
cultures sont déçus et les débouchés demeurent finalement limités. Le scandale naissant du chloredécone limite l'essor de filières de qualité comme l'agriculture biologique. Surtout la filière de la banane perd une grande partie de ses subventions. Depuis 2006, les négociations engagées par l'Union européenne, les États-Unis et certains pays d'Amérique latine dans le cadre de l'Organisation Mondiale du commerce ont conduit l'Union Européenne à libéraliser son marché et à réformer ses organisations communes des marchés pour la banane et le sucre. La filière sucre a ainsi abaissé de $36 \%$ le prix de son sucre dès 2009 .

Malgré le plan de modernisation 2007-2011 de nombreux signes de faiblesse apparaissent comme les nombreuses parcelles abandonnées, les 3000 pertes d'emplois depuis 2003 et les inquiétantes déclarations concernant la contamination des sols par les pesticides ainsi que le champignon cercosporiose noir qui affecte les rendements et entraîne un murissement accéléré du fruit nécessitant des traitements chimiques importants.

11 Le cyclone Dean atteint la Guadeloupe déjà fortement impactée par la conjoncture économique mondiale qui est en crise. À la hausse du prix du pétrole se rajoute la crise financière. Devant ces sombres perspectives qui ne permettent pas une projection positive post-cyclonique, la Guadeloupe et la Martinique connaissent un mouvement de protestation populaire de grande ampleur du mois de décembre 2008 au mois de mars 2009 avec le Collectif, Liyannaj Kont Pwofitasyon (LKP, alliance contre l'exploitation), un mouvement social unissant quarante-huit organisations, représentatives globalement des différents secteurs de la société. L'économie des îles a été paralysée nécessitant une réflexion des collectivités et de l'État pour trouver de nouveaux ressorts de développement.

12 À travers ces deux exemples, on peut remarquer une période de latence liée à la reconstruction, aux premières récoltes et à la reprise de la saison touristique. Cependant, si en 1989, Hugo a contribué à renforcer le processus de modernisation économique et sociale, en 2007, Dean a accéléré la déprise économique contribuant au malaise social et politique qui marque la fin de l'année 2008. Finalement le même schéma apparaît avec le passage d'Irma à Saint-Martin qui a déstabilisé une société et une économie insulaire qui présentaient déjà des éléments de crise : friches hôtelière, tensions sociales... (Redon, 2006).

\section{Les mesures adaptatives du bâti et des fonctions littorales en Guadeloupe : "l'avenir ne se prévoit pas, il se prépare » (Maurice Blondel, 1957)}

13 La résilience territoriale dépend de la capacité d'adaptation des individus qui varie et se modifie en fonction de la culture, de l'âge, de la situation familiale, de la profession (illustration 1). Cette capacité d'adaptation est aussi facilitée par le niveau de vie et dépend des politiques publiques. Enfin la maitrise et la connaissance des milieux de vie constituent aussi des facteurs importants (Quenault, 2013). 
Illustration 1 - Capacité d'adaptation au changement climatique dans un contexte d'augmentation de l'intensité et du nombre de cyclones

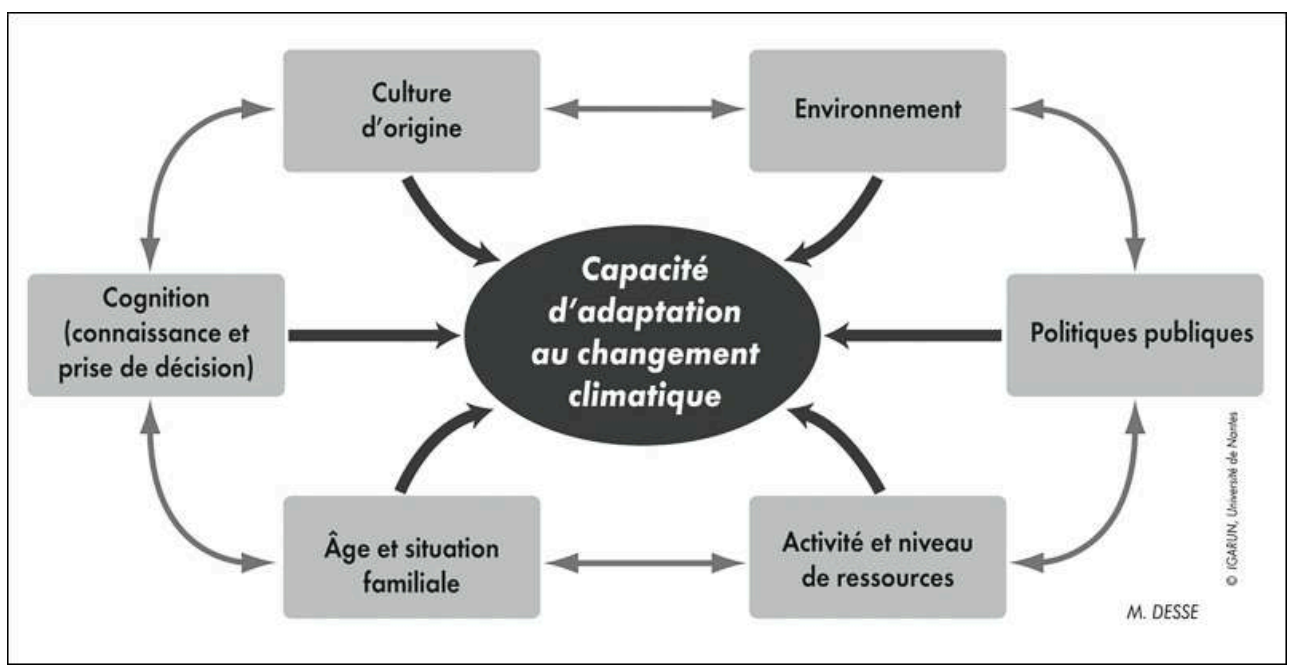

\section{L'adaptation des habitants}

Quand les maisons de première ligne ne sont pas détruites par la submersion induite par les houles cycloniques ou par arrêté municipal, les résidents de bord de mer s'adaptent à la menace de submersion marine. Le renforcement de la façade des cases en bois par un mur en parpaing apparait comme une première initiative. Avec le temps, c'est l'ensemble des murs qui sont remplacés, parfois un mur ou un enrochement protègent également la base des fondations.

Lorsque les habitants ont les moyens financiers nécessaires, la construction d'un étage apparaît aussi comme un moyen efficace de protection (illustration 2). L'ensemble de cette autoconstruction de protection peut transformer un front de mer et oblitérer parfois complètement l'ouverture sur la plage qui expliquait à l'origine l'installation des premières familles de marins-pêcheurs.

Pour les plus modestes, des vantaux métalliques qualifiés d'anti-cycloniques, permettent l'obturation des ouvertures et des façades en bois le temps du passage du cyclone. D'autres enfin s'en remettent à la providence ou à la chance. 


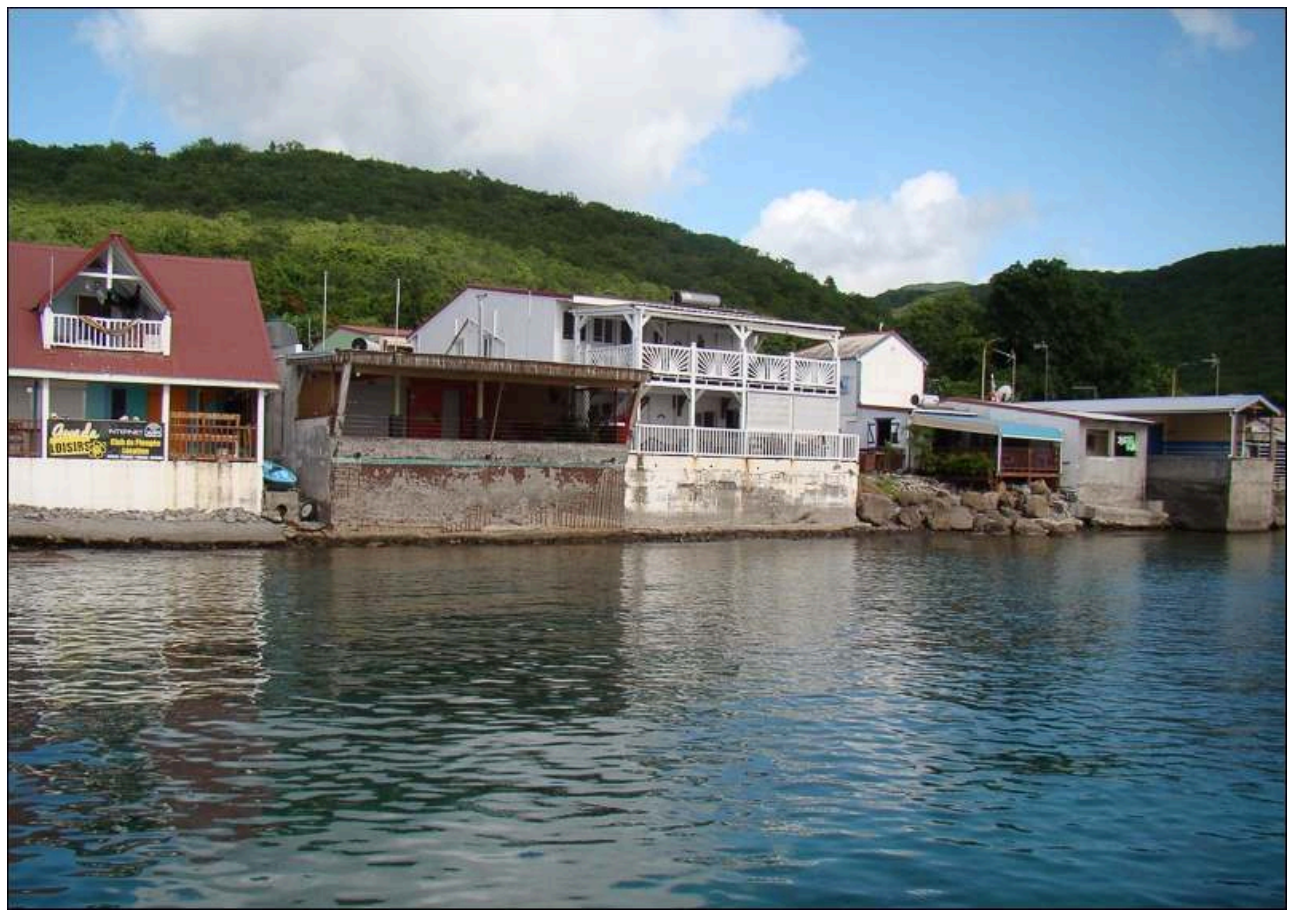

Cette photographie illustre les méthodes d'autoprotection des maisons par mur en béton et par un étage. En 1997, il ne restait de la plage initiale qu'un liseré de galets de 1,5 m de large. En 2018, la plage a disparu depuis une quinzaine d'années.

Auteur : M. Desse, 2018

L'abandon volontaire des fronts de mer par les habitants permanents constitue une autre forme d'adaptation. Dans les anciens quartiers d'habitat spontané de marinspêcheurs, comme à Baille-Argent (commune de Pointe-Noire), les femmes âgées, sont aujourd'hui majoritaires. Quelques nouveaux habitants souvent modestes occupent parfois en location, les vieilles cases. Lorsque les parents décèdent, les enfants préfèrent construire ailleurs, vers les villes ou en hauteur dans les jardins familiaux que possède un grand nombre de familles et qui participaient à la pluriactivité nécessaire à la survie des pêcheurs. Dans d'autres cas, les vieilles maisons sont louées pour la location touristique ou la restauration. À Baille-Argent, la route attire les services au détriment du bord de mer devenu un espace en marge protégé par un enrochement (illustration 3). 
Illustration 3 - Le village de pêcheur de Baille-Argent

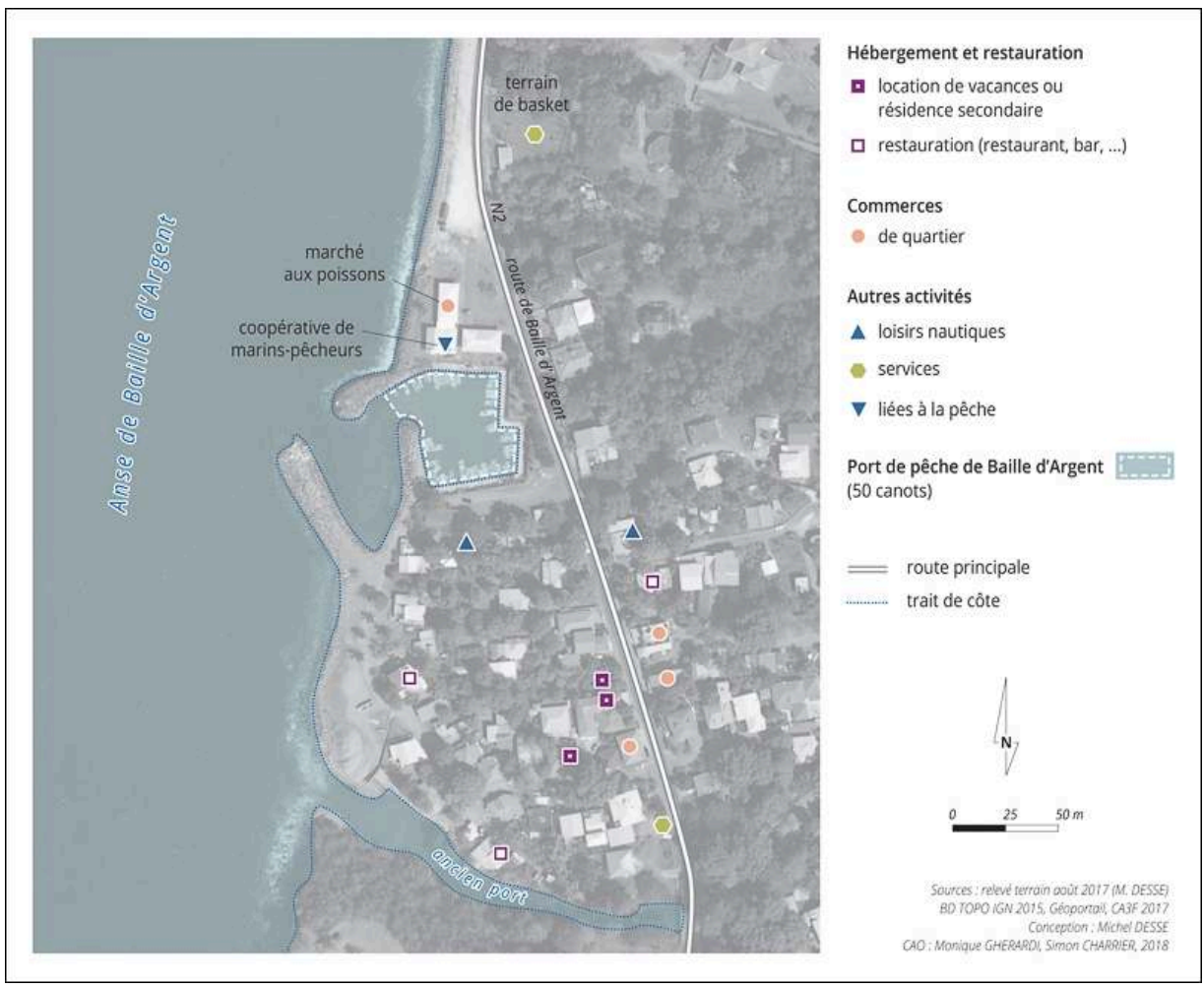

Autrefois tourné vers son vieux port, il s'organise aujourd'hui autour de la N2. Le bord de mer n'est plus un lieu de vie, il est devenu un terrain vague bordant l'enrochement de protection.

\section{Le rôle des politiques d'urbanisme et de prévention des risques}

Depuis le cyclone Hugo en 1989, les municipalités et les services de l'État prennent en compte le risque de houle cyclonique qui menace à chaque hivernage les habitants du littoral. En côte-sous-le-vent de Guadeloupe, les municipalités restructurent les quartiers spontanés du front de mer construits depuis les années 1950 et recasent les habitants dans des logements évolutifs sociaux en arrière du littoral. Le quartier de Pigeon à Bouillante (Guadeloupe) a ainsi bénéficié de cette procédure. Les résidents ont été relogés dans les logements évolutifs sociaux de Desmarais et des Galets. On retrouve les mêmes opérations à Ferry (commune de Deshaies) et à l'Anse-Guyonneau à PointeNoire (Desse et Saffache, 2005).

Dans les bourgs littoraux qui se tournent vers le tourisme, la principale tension réside entre le maintien du lieu qui offre une situation économique attractive et la sécurité des personnes. À Deshaies, le POS des années 1990 présente ainsi une volonté manifeste d'une meilleure intégration du front de mer avec l'embellissement des façades et la plantation d'arbres. La construction d'un deck le long de la plage comme promenade est même envisagée (Gaspard, 1998). Des aménagements pour les marins pêcheurs sont aussi prévus à la suite de concertation avec les professionnels. Le schéma de restructuration du bourg prévoit 4 scénarios : le laissé faire, la destruction des maisons de bord de mer remplacées comme à Ferry par un enrochement, la construction d'un mur pour protéger les maisons en haut de plage ou encore celui qui prévaut depuis 2013, le libre choix aux propriétaires de se protéger en renforçant les murs des façades 
donnant sur la mer (POS de Deshaies). Le remplacement des habitations de bord de mer par des commerces et des établissements de restauration pouvant être facilement évacués, apparaît comme une mesure favorisant ce contrôle des enjeux. Ce passage d'un front de mer investi par les marins-pêcheurs, aux activités de loisir et de tourisme, est devenu nécessaire à cause de la succession de submersions marines. Il a aussi été facilité par le déclin des activités halieutiques en partie liées à la surpêche des eaux côtières et au vieillissement de la profession et d'autre part aux financements des structures touristiques locales à partir des années 1990.

L'État et les collectivités territoriales sont aussi des acteurs de cette requalification littorale. Le littoral juridique guadeloupéen dépend de la zone des 50 pas géométriques qui couvre une largeur de $81,20 \mathrm{~m}$. Cette largeur est calculée à partir de la ligne des plus hautes marées. En 1955, afin de favoriser le développement économique, un décret reclasse l'ensemble de la zone des 50 pas géométriques dans le domaine privé de l'État. Les collectivités locales et les particuliers avaient la possibilité pendant un an de faire valider leurs droits d'occupation. En 1986, la nécessité de renforcer la protection du littoral a conduit le législateur à réintégrer cette zone dans le domaine public de l'État. Toutefois la loi littorale ainsi que la loi du 30 décembre 1996 ouvrent sous conditions des possibilités d'acquisition foncière aux occupants de cette zone (Chadenas et al., 2016).

Depuis 1996, ces quartiers littoraux qui ont accueilli le trop plein des campagnes sont reconnus par l'État. La loi du 30 décembre 1996 relative à l'aménagement, la protection et la mise en valeur de la zone dite des « 50 pas géométriques » dans les départements d'Outre-mer conforte la loi littorale de 1986 en limitant les constructions aux zones urbanisées ou d'habitat diffus (Desse, 2013). Les occupants doivent prouver que la parcelle était construite avant le $1^{\mathrm{er}}$ janvier 1955 et occupée à usage d'habitation principale ou de bâtiment professionnel pour obtenir une cession à titre onéreux en fonction de la valeur vénale du terrain nu. La superficie du terrain est plafonnée à $500 \mathrm{~m}^{2}$.

La loi $n^{\circ}$ 2010-788 dite "Grenelle 2 » ambitionne d'arrêter le flux des constructions illicites et d'accélérer le processus de régularisation des occupants sans titre de la zone. Enfin la loi n 2011-725 sur l'habitat informel et indigne dite loi " Letchimy » en 2011, offre aux acteurs publics la possibilité de conduire des opérations d'aménagements sur des quartiers d'habitat informel soumis à de forts risques naturels (Chadenas et al., 2016 ; illustration 4$)$. 
Illustration $4 a$ - Avis de recherche d'un éventuel propriétaire

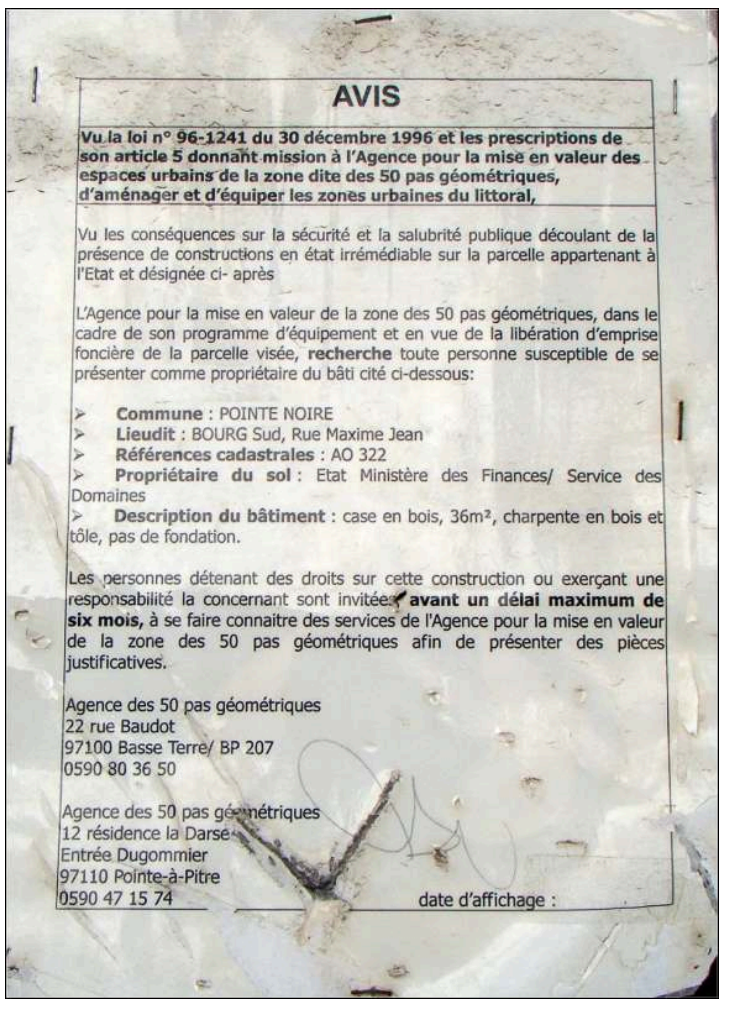

\section{Illustration 4b - État des cases traditionnelles}

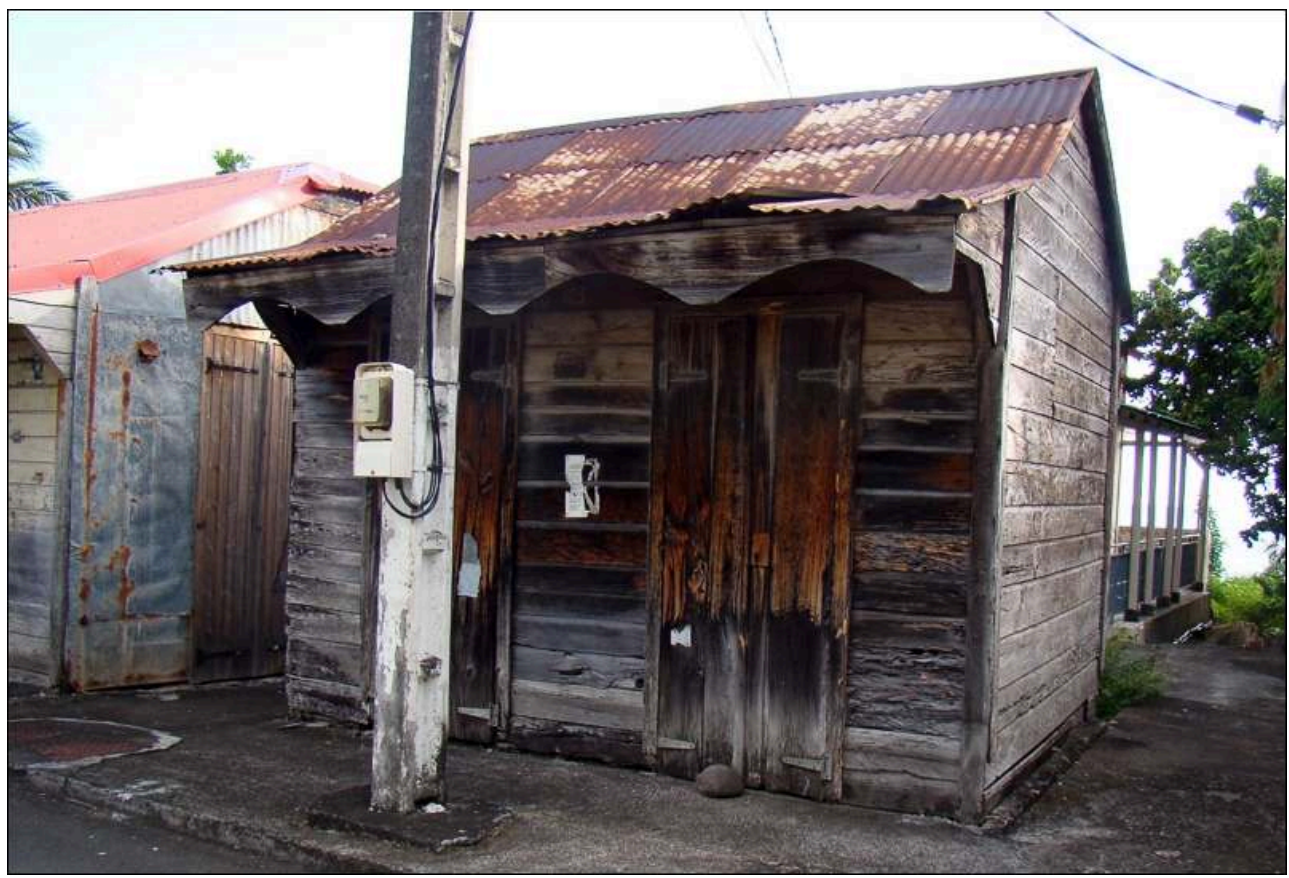

Cases menacées de destruction par l'agence des 50 pas géométriques dans le cadre de la loi «Letchimy » en 2011, dite de l'habitat informel et indigne.

Auteur: M. Desse, 2018. 


\section{La requalification des fronts de mer touristiques entre adaptation et anticipation : les exemples de Deshaies et de Sainte-Anne}

En 1982, la commune de Deshaies compte 3485 habitants (3 271 en 1961), et connait une certaine léthargie marquée par la stagnation de sa population (3 476 habitants en 1990). Le bourg regroupe l'essentiel de la population qui se localise aussi dans des quartiers (sections) littoraux à Ferry au Sud, Ziotte, la Perle, Rifflet et Grande Anse au nord ainsi que sur les pentes (Caféières et Leroux). L'appareil commercial est réduit à quelques commerces de proximité, quelques marchandes de fruits et légumes pays. Afin de s'adapter aux difficultés économiques après la crise des cultures fruitières et du café et cacao propre à la Côte-sous-le-vent, de nombreux habitants sont polyactifs, pêcheurs, agriculteurs et trouvent dans le bâtiment un complément d'emploi dans un contexte ou le chômage est important (38,5\% en 1990). On compte encore en 1986, 54 pêcheurs déclarés aux affaires Maritimes pour une centaine de saintoises et canots. Quatre hôtels, deux restaurants et un commerce de souvenirs profitent de l'arrivée des touristes. Deshaies est vantée par les guides touristiques et attire les bateaux de plaisance au mouillage et quelques artistes, en particuliers Robert Charlebois et Coluche. Trois ans après les destructions occasionnées par le cyclone Hugo, le décor a peu changé. En 1992, Deshaies reste un village enclavé au nord de la Basse-Terre et occupe la $30^{\mathrm{ème}}$ place sur 35 communes en Guadeloupe dans la hiérarchie fonctionnelle des centres qui prend en compte les commerces et les services.

Il faut attendre les années 1995 pour que l'on assiste au démarrage démographique (4 039 habitants en 1999 et 4114 en 2015) et à l'essor des activités de tourisme, locations, commerces, restaurants, hôtels, clubs de plongée, excursions en mer se multiplient. Paradoxalement, alors que la plage qui bordait la première ligne de maisons disparait, l'attractivité touristique du bourg se renforce par son appareil commercial centré sur le tourisme et le loisir essentiellement localisé en front de mer (illustration 5). Peu à peu, les habitants du bord de mer ont vendu, loué ou transformé leurs locaux en restaurants, locatifs, magasins de souvenirs, club de plongée. Depuis Hugo, chaque cyclone a joué son rôle et décidé les habitants les plus exposés à se reloger ailleurs. Les premiers habitants sont peu nombreux et âgés, si les enfants conservent les vieilles maisons, c'est avant tout pour leur situation en front de mer et la possibilité de les louer à de nouveaux entrepreneurs du tourisme. 
Illustration 5 - Le bourg de Deshaies en 2018

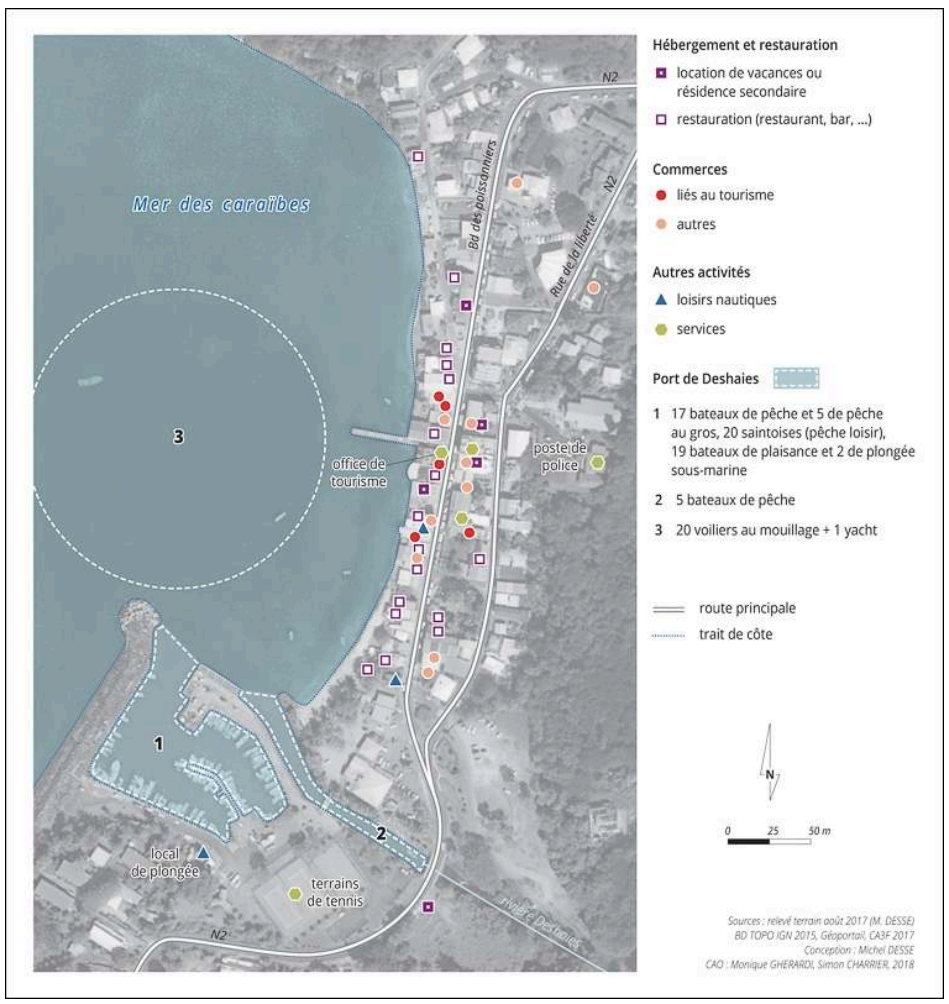

Ce dernier est tourné vers les activités touristiques, où les activités commerciales liées au tourisme et les offres de loisirs nautiques occupent l'essentiel des maisons du front de mer.

La commune de Sainte-Anne située en Grande-Terre, participe à l'aire urbaine et à la zone touristique de la Grande-Terre depuis les premiers plans quinquennaux de développement du tourisme. Elle connaît une progression régulière de sa population avec 12481 habitants en 1961, 13826 en 1982, 20410 en 1999 et 24379 en 2015. Depuis les années 1980, elle apparaît comme une commune vivant du tourisme et profitant par sa position du dynamisme de Gosier et de Saint-François qui toutes les deux ont connu des investissements conséquents et des installations de grands hôtels internationaux. Avec l'installation du club Méditerranée, très vite restaurants de bord de mer et boutiques proposant des articles touristiques se développent à proximité du Club Med, mais aussi dans le bourg. Durant les années 1980, les hôtels haut de gamme, à capitaux insulaires se sont aussi implantés, entraînant le secteur de la restauration, la location de voitures, les entreprises d'excursion en mer autour des îlets de Petite et GrandeTerre. En 1989, l'agglomération a été très fortement impactée par le cyclone Hugo, puisque la submersion marine a affecté les habitations sur une centaine de mètres. Dans la partie rurale de la commune, les dégâts ont accentué la perte du petit paysannat. La tertiarisation des activités par le tourisme et l'économie résidentielle apparaît comme une opportunité. À la suite, ce village de pêcheurs a accueilli des résidences secondaires, des résidents aisés travaillant dans l'agglomération de Pointeà-Pitre, des locations pour les Guadeloupéens comme pour les touristes métropolitains. En 2018, le front de mer de l'agglomération propose toutes les fonctionnalités d'une station balnéaire tout en conservant un décor paysager de qualité (illustrations $6 \mathrm{a}$ à $6 d)$. 
$\mathrm{Au}$ centre, les plages connaissent une densité des équipements et des fonctions : jeux de plage, cafés et restaurants de plage, marché artisanal proposant des productions locales et divers services comme les excursions en mer. Un second marché alimentaire, accueillant aussi des vendeurs d'articles touristiques, intériorise cette centralité. De part et d'autre, le long du front de mer, les magasins de souvenirs et de vêtements de plage, de restauration rapide remplacent peu à peu l'appareil commercial traditionnel (épicerie) et l'habitat ancien... les petites cases ne répondant plus aux normes de confort. Ces dernières ont aussi parfois connu des atteintes du fait des houles cycloniques, entrainant parfois un simple dérangement (eau, sable) parfois de réels endommagements. Un troisième marché artisanal assure la jonction entre la zone hôtelière et le bourg. Les activités et les aménagements liés à la pêche, qui constituaient l'essentiel de l'occupation du front de mer, ont été regroupés à la marge du bourg, entre le Club Méditerranée et le bourg. À la zone hôtelière qui occupe l'ouest de la plage, répondent les petits hôtels, les entreprises proposant une dizaine de bungalows, les résidences secondaires qui s'étendent en bordure de mer (Desse et al., 2018). Ponctuellement en fin de semaine, à l'occasion d'évènements festifs, ces espaces de bord de mer connaissent des afflux de commerces ambulants (tatouages, vente de repas antillais, petit artisanat). Ce passage d'un lieu tourné vers la pêche aux fonctions récréatives et touristiques est pleinement approprié par les habitants qui y développent toutes les pratiques d'une plage standard: baignade, natation, aquagym, longe côte, promenade sans oublier les pratiques locale des repas familiaux en bord de mer.

\section{Illustration 6a - Plan de localisation}

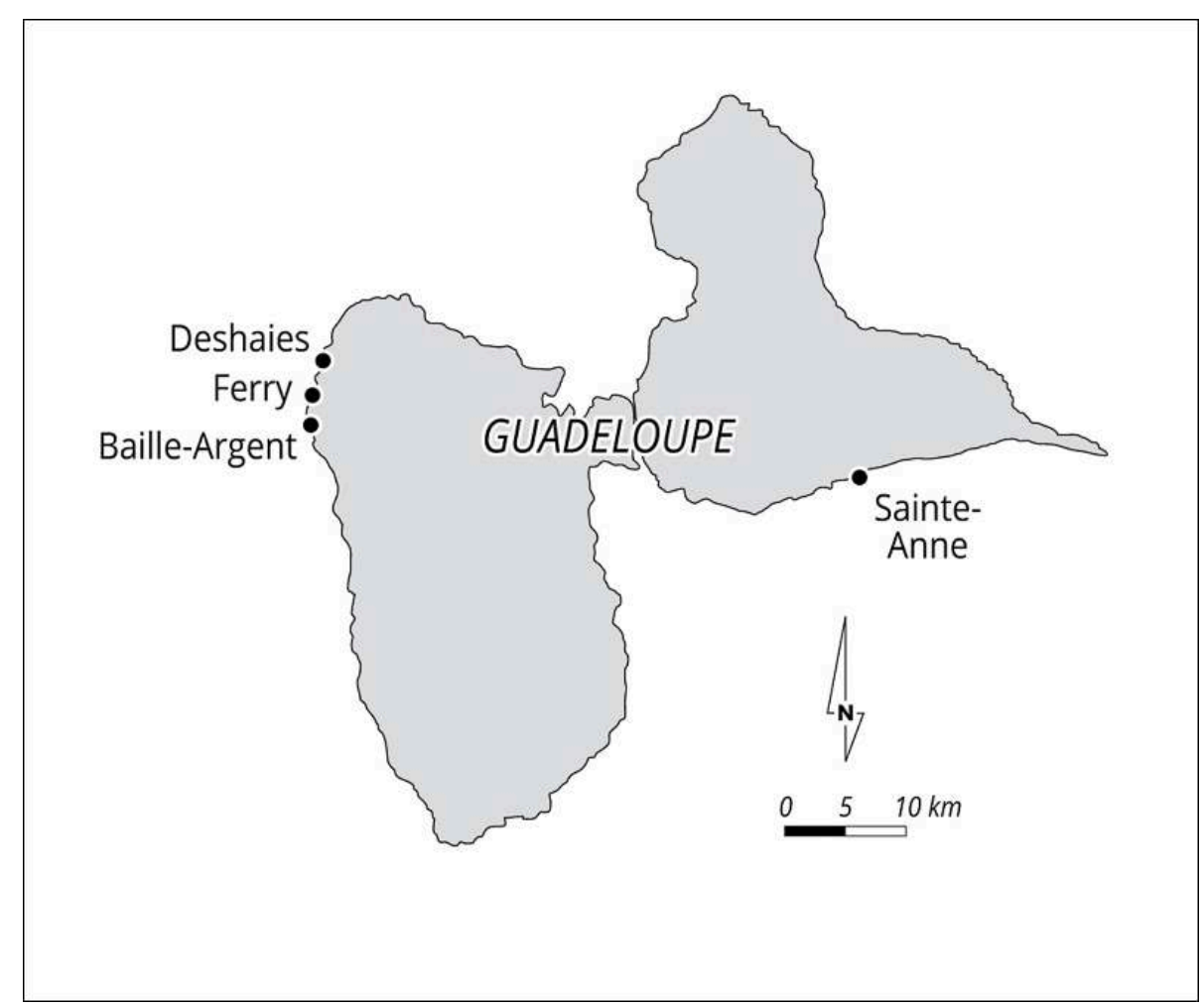


Illustration 6b - Bourg de Sainte-Anne par secteur (Ouest, Centre et Est)

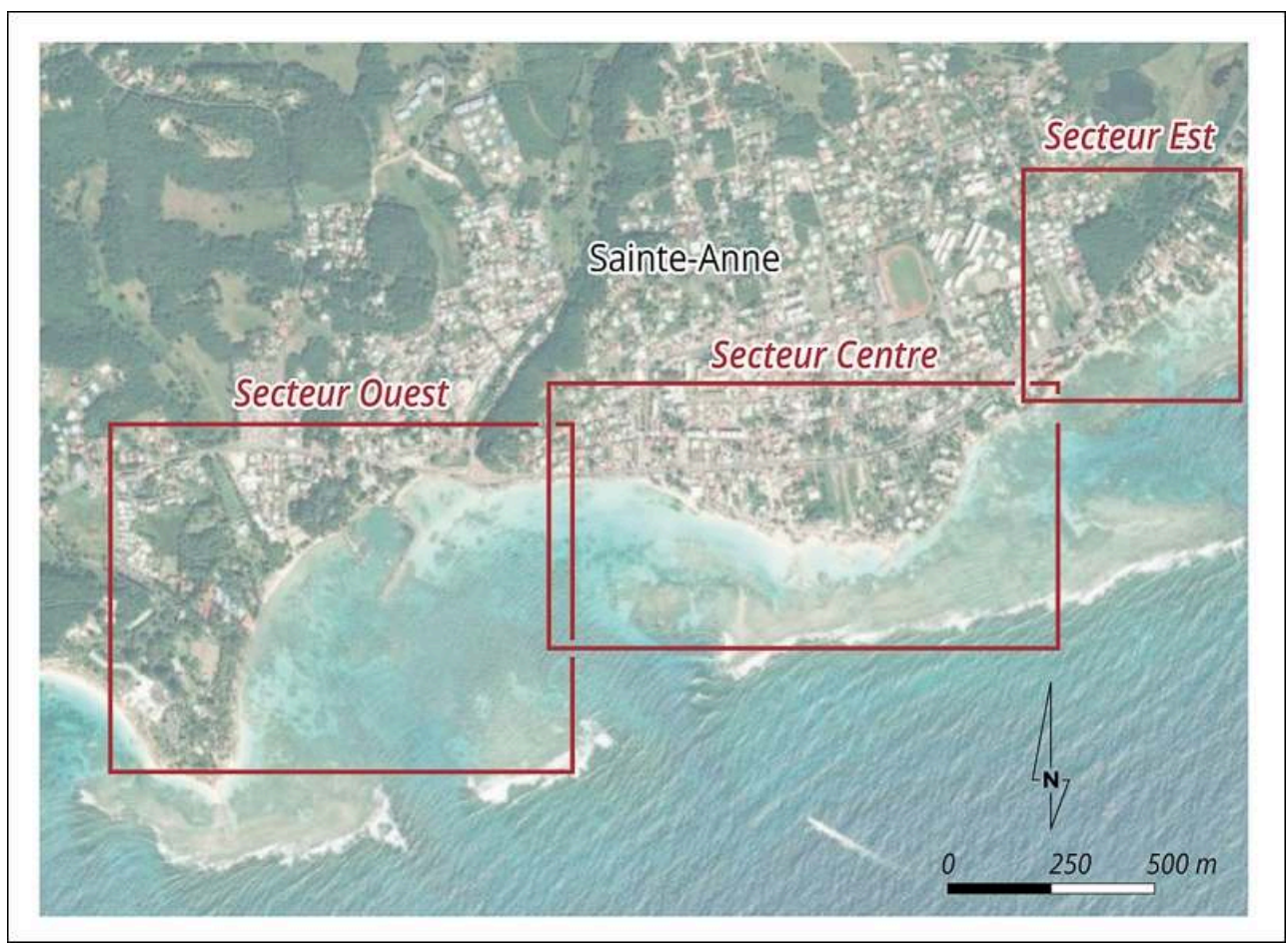

Illustration 6c - Bourg de Sainte-Anne secteur Ouest

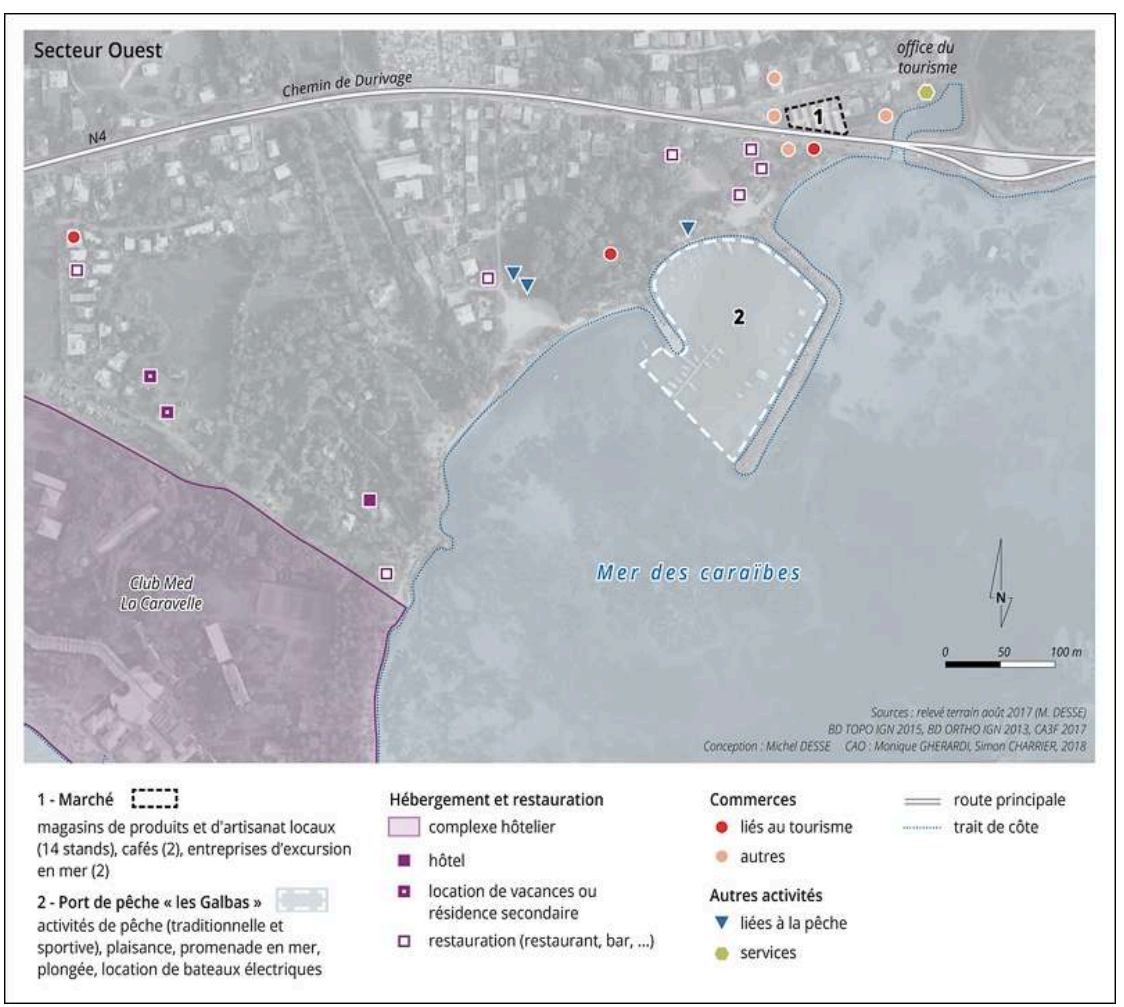




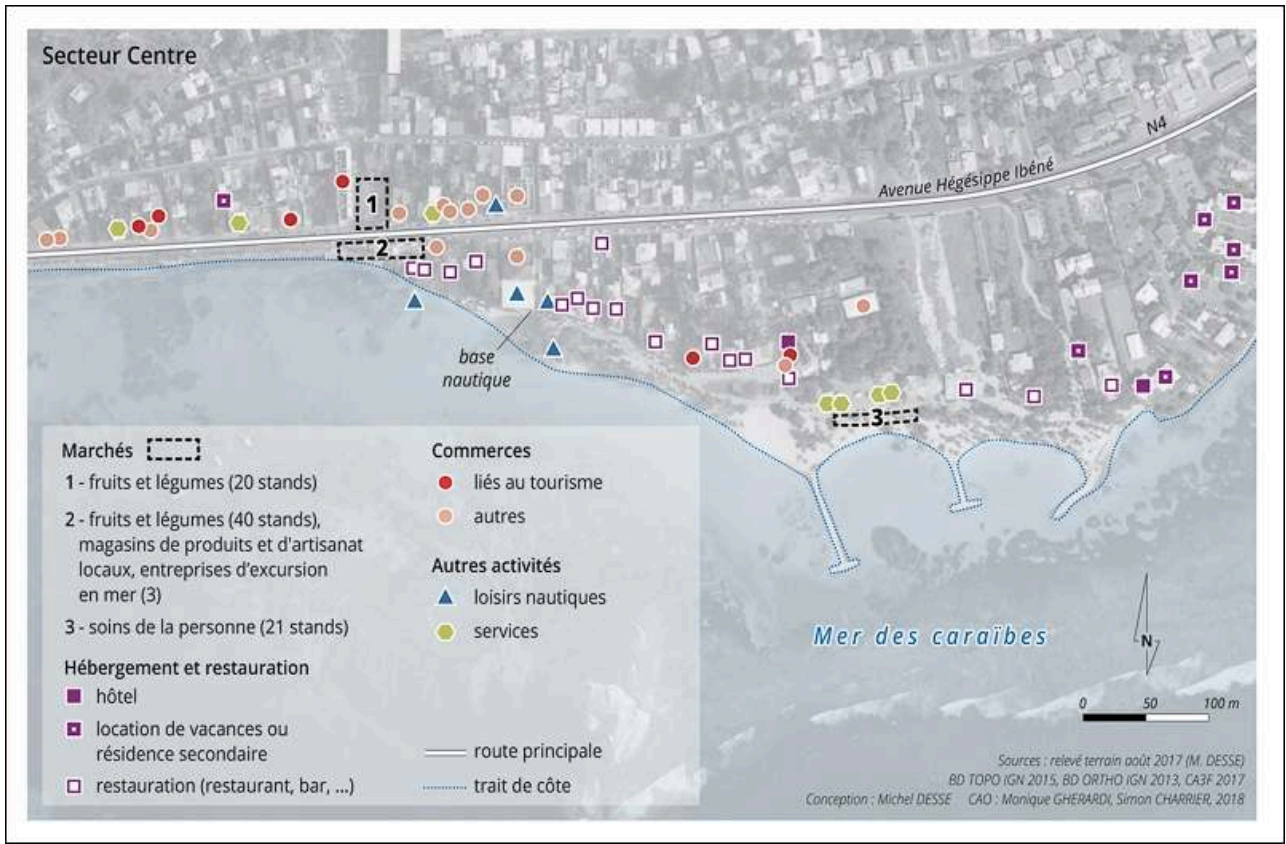

Illustration 6e - Bourg de Sainte-Anne secteur Est

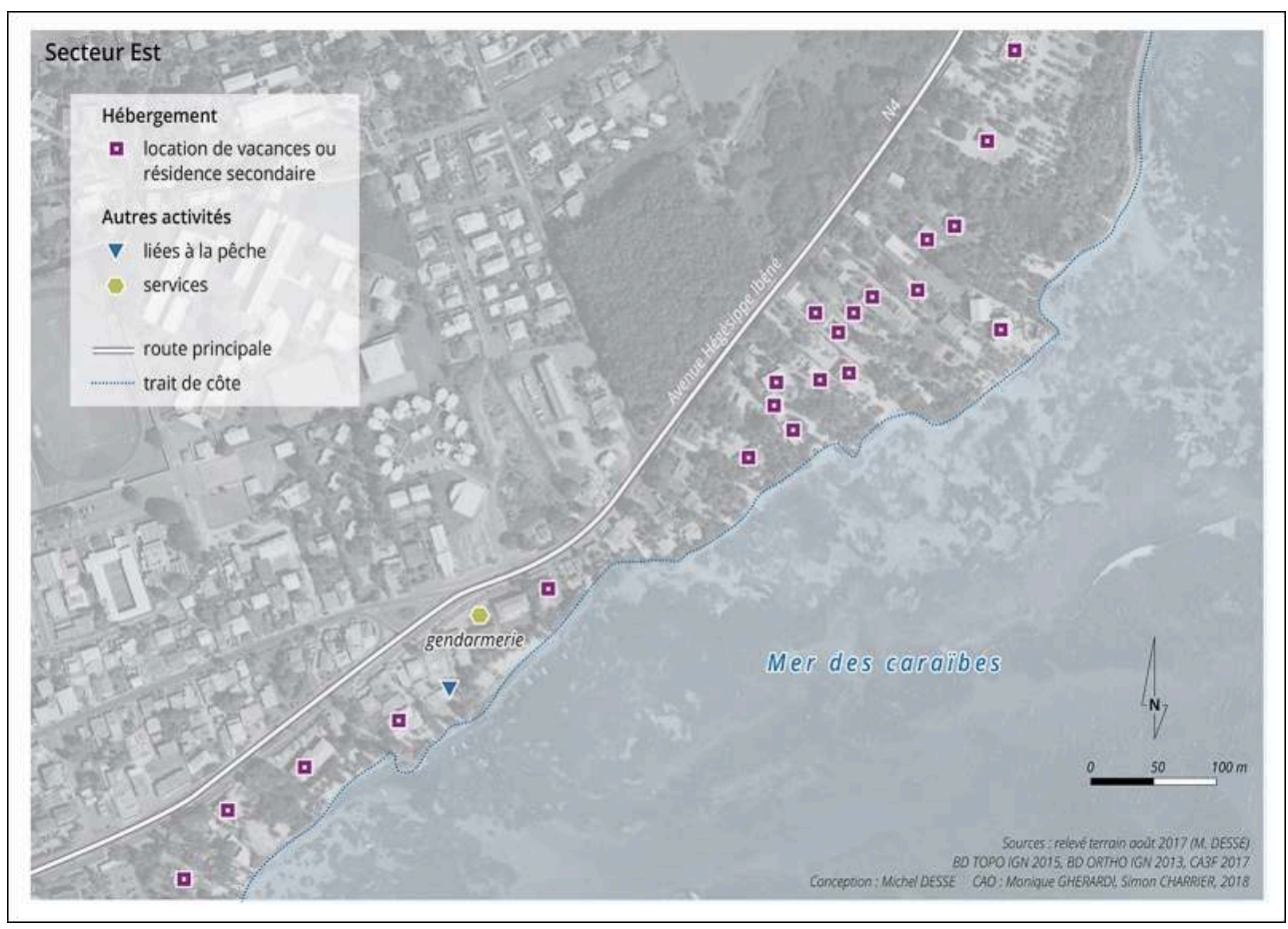

Les activités touristiques se déploient sur le front de mer, par la requalification de l'habitat et des fonctions traditionnelles liées à la pêche par des commerces permanents ou forains spécialisés vers le tourisme et l'offre de loisir.

$\mathrm{Au}$ travers de ces deux communes de Guadeloupe, deux stratégies apparaissent. La première consiste à détruire les constructions de bord de mer et souvent de renforcer les ouvrages d'art par des enrochements qui tendent à se multiplier, la seconde dans certains sites attractifs, à maintenir les constructions si elles sont affectées aux 
fonctions touristiques. Ainsi en cas de cyclone, les dégâts humains seront limités. Pour autant, le littoral ne perd pas sa valeur bien au contraire, et la situation « les pieds dans l'eau » constitue un attrait dans les domaines de la restauration, des loisirs de plages, du commerce balnéaire.

De même les parcelles avec vue mer et donnant sur la mer reprennent de la valeur pour les promoteurs immobiliers et les habitants ; marque de leur attachement au lieu. Alors que l'Est du littoral de Sainte-Anne connaît une très forte érosion, il n'a pourtant pas été affecté par une houle cyclonique depuis 1989, dès que la saison cyclonique s'estompe, les projets de construction reprennent.

\section{Conclusion}

En fonction du contexte économique et politique, une société est plus ou moins résiliente parce qu'elle ne présente pas, à 15 ans d'écarts, les même caractéristiques même sur un territoire si particulier et étroit qu'une île tropicale.

De même, un état, une région peuvent faire preuve de résilience en 1989 à la suite du passage du cyclone Hugo et impulser une nouvelle perspective économique et sociétale car la dynamique était déjà engagée. Il peut aussi, parce qu'il est lui même atteint par une crise qui le dépasse comme les crises de 2007 et de 2008, manquer de ressort et ne pas trouver les leviers du développement territorial, qu'ils soient économiques, sociaux et sociétaux, nécessaires à une société résiliente. Ces deux territoires montrent l'importance du levier sociétal qui relève du projet commun et qui permet de dépasser le choc initial qui peut mener à l'apathie. À l'échelle locale sur les fronts de mer, les initiatives d'adaptation sont nombreuses et se déploient. Loin d'être une marge marquée par le retrait des activités et des habitants, les fronts de mer connaissent une requalification orientée vers les activités de tourisme et de loisir et finalement la valeur foncière augmente du fait des avantages économiques liés à la plage et à la vue sur la mer.

\section{BIBLIOGRAPHIE}

Berger G., 1957. Sciences humaines et prévision. La Revue des Deux Mondes, n 3, p. 417-426.

Chadenas C., Rollo N., Desse M., 2016. Les 50 pas géométriques dans les territoires ultramarins. Les Cahiers Nantais, $\mathrm{n}^{\circ}$ 2, Igarun, p. 43-52.

Desse M., Saffache P., 2005. Les littoraux antillais : des enjeux de l'aménagement à la gestion durable. Paris, Ibis Rouge, $116 \mathrm{p}$.

Desse M., 2011. Les pressions anthropiques, les mesures de protection et les défis de gestion participative en Guadeloupe et à la Martinique. In Chouinard O., Baztan J. et Vanderlinden J.-P. (dir.), Zones côtières et changement climatique. Presses de l'Université du Québec, p. 165-166.

Desse M., 2013. Les îles de la Caraibe, enjeux et perspectives. L'Harmattan, 290 p. 
Desse M., Rodne Jeanty M., Gherardi M., Charrier S., 2018. Le tourisme dans la Caraïbe, un moteur du développement territorial, IdeAs [En ligne], $\mathrm{n}^{\circ}$ 12. URL: http://journals.openedition.org/ideas/ 4239 - DOI: https://doi.org/10.4000/ideas.4239

Flanquart H., 2016. Des risques et des hommes. PUF, 350 p.

Gaspard G., 1998. Variation du niveau marin et gestion du littoral à Deshaies (Guadeloupe). Université des Antilles et de la Guyane, 120 p.

Lassailly-Jacob V., Desse M., 2010. Migrations et vulnérabilités. Eleveurs sahéliens confrontés aux sécheresses et populations des littoraux antillais face aux cyclones. In Colloque international Les migrations et les écosysytèmes environnementaux : les réfugiés de l'environnement. Rabat, Université Mohamed V, p. 79-101.

Pagney Benito-Espinal F., 1998. Croissance démographique et environnement : approche et perspectives en Guadeloupe. Les Cahiers d'Outre-Mer, n² 201, p. 3-20.

Pagney Benito-Espinal F., 2002. Les risques de tempêtes et d'ouragans en Martinique et en Guadeloupe. Analyse comparée. In Burac M., Desse M. (dir.), Actes du colloque Les Départements Français d'Amérique à l'aube du XXI ${ }^{e}$ siècle : leur développement économique et social. Paris, Karthala, p. 311-326.

Redon M., 2006. Saint-Martin/Sint-Maarten, une petite île divisée pour de grands enjeux. Les Cahiers d'Outre-Mer, $\mathrm{n}^{\circ} 234$, p. 233-266.

\section{NOTES}

1. Depuis 1966, on dénombre huit cyclones en Guadeloupe (Inez en 1966, David en 1979, Hugo en 1989, Luis en1995, Marilyn en1995, Lenny en 1999, Maria en 2017 et Isaac en 2018)

2. Les DCP sont constitués d'un cordage de $3000 \mathrm{~m}$, fixé à un corps-mort et à une bouée. Entre 50 mètres et la surface, une série de vieux filets, de bâches constituent autant de supports permettant la création de petits écosystèmes qui attirent à leur tour les gros pélagiques (marlins, thons, espadons). Les premiers DCP ont été exploités en Guadeloupe en 1980 pour se diffuser ensuite dans la Caraïbe.

\section{RÉSUMÉS}

La Guadeloupe est soumise aux cyclones qui génèrent des dégâts importants. En fonction du contexte économique local et mondial, les cyclones engendrent des ruptures positives ou négatives pour les sociétés et les économies insulaires. Les formes adaptatives demeurent les plus nombreuses à toutes les échelles du territoire à l'initiative des collectivités territoriales, des filières de production comme des habitants. Les fronts de mer, moins habités, demeurent cependant le support d'activités à grande valeur ajoutée souvent en lien avec le tourisme à grande valeur ajoutée.

Guadeloupe is subject to hurricane that generate significant damage. Depending on the local and global economy, cyclones create positive or negative disruptions for island societies and 
economies. Adaptive forms remain the most numerous at all scales of the territory at the initiative of local authorities, production sectors and inhabitants. The sea fronts, less inhabited, however, remain the support of activities often related to tourism with high added value.

\section{INDEX}

Keywords : hurricane, Guadeloupe, storm surge, adaptability, spatial amendment

Mots-clés : cyclone, Guadeloupe, submersion marine, adaptation, requalification spatiale

Thèmes : Sur le Champ - Sur le Terrain

\section{AUTEURS}

\section{MICHEL DESSE}

Michel Desse, michel.desse@univ-nantes.fr, est professeur des Universités à l'Université de Nantes et membre de l'UMR 6554 Littoral, Environnement, Télédétection, Géomatique (LETG). Il a récemment publié :

- Desse M., Nordin C., 2015. Saint-Barthélemy: en rik karibisk ö som utmärker sig. Sverige utanför-Swensk makt och dess spar i utlandet. Svenska Sällskapet för Antropologi och geografi, p. 85-102.

- Desse M., El Bouzidi A., Peyrusaubes D., 2017. Perception et adaptation au changement climatique dans le Gharb atlantique dans un contexte de mutations socioéconomiques dynamiques. Les impacts du changement climatique sur les littoraux en Afrique. Territoire d'Afrique, $\mathrm{n}^{\circ}$ 10, p. 100-115.

- Lemee C., Guillard M., Fleury-Bahi G. et al., 2019. What meaning do individuals give to coastal risk? Contribution of the social representation theory. Marine Policy.

\section{MONIQUE GHERARDI}

Monique Gherardi, monique.gherardi@univ-montp3.fr, est ingénieur d'études et membre de l'UMR GRED - Université Paul Valéry Montpellier 3. Elle a récemment publié :

- Leone F., Komorowski J., Gherardi-Leone M. et al., 2019. Integrating spatial accessibility in the design of volcano evacuation plans in the French West Indies (Guadeloupe and Martinique). J. Appl. Volcanol., vol. 8, n 8. DOI: https://doi.org/10.1186/s13617-019-0089-1

- Rey T., Leone F., Candela T. et al., 2019. Coastal Processes and Influence on Damage to Urban Structures during Hurricane Irma (St-Martin \& St-Barthélemy, French West Indies). Journal of Marine Science and Engineering [En ligne], vol. 7, n 7.DOI: https://doi.org/10.3390/jmse7070215 - Diop A., Gherardi M. (dir.), 2019. Rivages et horizons. Hommages au géographe Jean-Marie Miossec. Paris, Edition L'Harmattan, 470 p.

\section{SIMON CHARRIER}

Simon Charrier, simon.charrier@univ-nantes.fr, est cartographe à l'Université de Nantes IGARUN. Il a récemment publié :

- Desse M., Charrier S., 2017. La grande plaisance, un secteur économique en plein essor. Études caribéennes [En ligne], $\mathrm{n}^{\circ}$ 36. URL : http://etudescaribeennes.revues.org/10562

- Pottier P., Ovono M. et al., cartographie de CHARRIER S., 2017. Les régions littorales du GabonÉléments de réflexion pour une planification stratégique du territoire. LETG-Géolittomer/ RapondaWalker, $417 \mathrm{p}$. 
- Desse M., Rodne Jeanty J., Gherardi M., Charrier S., 2018. Le tourisme dans la Caraïbe, un moteur du développement territorial. IdeAs [En ligne], $\mathrm{n}^{\circ}$ 12. URL : http://journals.openedition.org/ ideas/4239 - DOI: https://doi.org/10.4000/ideas.4239 